\title{
A Case of Recurrent Chest Pain With Reversible Left Ventricular Dysfunction and ST Segment Elevation on Electrocardiogram
}

\author{
Hiroyuki YaOITA, ${ }^{1} \mathrm{MD}$, Takayoshi YAMAKI, ${ }^{1} \mathrm{MD}$, Hiroyuki MizUGAMI, ${ }^{1} \mathrm{MD}$, \\ Nobuo SaKamoto, ${ }^{1} \mathrm{MD}$, Keiichi KaWAmura, ${ }^{1} \mathrm{MD}$, Yuichi UJIIE, ${ }^{1} \mathrm{MD}$, \\ Kazuhira MAEHARA, ${ }^{1}$ MD, and Yukio MARUYAMA, ${ }^{1}$ MD
}

\begin{abstract}
SUMMARY
There is a syndrome consisting of acute infarction-like symptoms and ECG findings, and transient left ventricular apical ballooning without epicardial coronary artery obstruction. A 67-year-old female admitted to our hospital because of severe anterior chest pain was diagnosed as having this syndrome. Since stenotic, spastic, or occlusive sites were not found in epicardial coronary arteries by emergency cardiac catheterization, we speculated coronary microvasculature involvement in the pathophysiology of the event. Four weeks later in a drug-free condition, there was no significant epicardial coronary vasospasm by intracoronary acetylcholine administration (IC-ACh). The average peak flow velocity (APFV) of the left coronary artery (LCA) was measured using the Doppler flow wire method. Under maximal dilatation of the epicardial LCA by intracoronary nitroglycerin administration, IC-ACh was again performed taking into consideration that the change in APFV in response to IC-ACh reflects a coronary microvascular response to it. In the nonischemic control subjects, basal APFV increased to $296 \pm 29 \%(n=24)$ of the basal value after IC-ACh. In this patient, although IC-ACh did not cause vasospasm in epicardial LCA, APFV was decreased to $54 \%$ of its basal value. After administration of a $\mathrm{Ca}$ antagonist and KATP opener, she had no chest symptoms and was discharged from the hospital. In 2003, she forgot to take her medication for 3 days and then experienced a sudden recurrence of the same type of attack. She started her medication again and her symptoms disappeared. Three weeks later, she underwent an assessment of the coronary microvascular response to ACh with medicine. Her APFV after ACh increased to $177 \%$ of the basal value. (Int Heart J 2005; 1: 147-152)
\end{abstract}

Key words: Ampulla, Cardiomyopathy, Ischemia, Microcirculation, Flow reserve, Ca antagonist, KATP opener

THERE have been reports ${ }^{1-7)}$ on subjects with acute myocardial infarction-like symptoms and ECG findings, and transient left ventricular apical ballooning

From ${ }^{1}$ the First Department of Internal Medicine, Fukushima Medical University, Fukushima, Japan.

Address for correspondence: Yukio Maruyama, MD, First Department of Internal Medicine, Fukushima Medical University, Hikarigaoka 1, Fukushima 960-1295, Japan.

Received for publication March 9, 2004.

Revised and accepted July 8, 2004. 
without coronary artery stenosis. The pathogeny of this phenomenon, which is sometimes called 'ampulla cardiomyopathy', has not been clarified ${ }^{4)}$ although catecholamine burden may be one of the causes in some patients. ${ }^{1-3,8)}$ Since stenotic, spastic, or occlusive sites are not found in epicardial coronary arteries in such patients, involvement of the coronary microcirculation in the pathophysiology of this syndrome may be plausible. ${ }^{4,7)}$ We experienced a female case who had recurrent chest pain attacks that were probably related to this syndrome. The clinical course and data for cardiac catheterization suggested the involvement of coronary microcirculatory disturbance in this patient's morbidity.

\section{Case Report}

A 67-year-old female was admitted for the first time to our hospital in October 1999 because of severe anterior chest pain. On admission, ST segments on ECG were elevated in $\mathrm{I},{ }_{\mathrm{a}} \mathrm{V}_{\mathrm{L}}$, and $\mathrm{V}_{2}$ through $\mathrm{V}_{6}$ leads by 0.2-0.4 $\mathrm{mV}$ (Figure 1). The chest pain was not relieved with sublingual nitroglycerin. The patient underwent emergency cardiac catheterization due to a diagnosis of acute coronary syn-
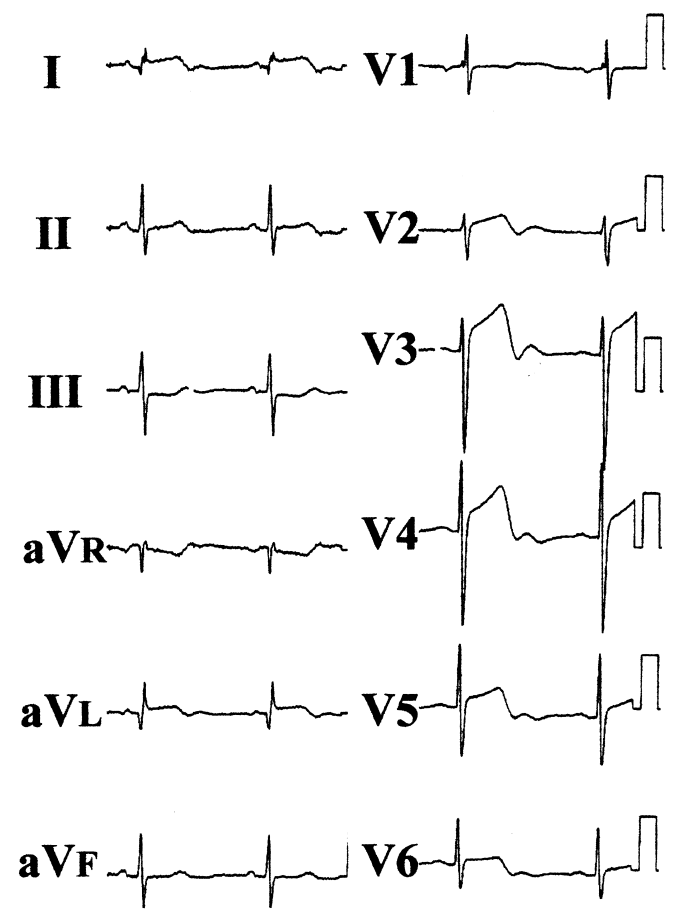

Figure 1. ECG on admission.

ST segments on ECG were elevated in $\mathrm{I},{ }_{\mathrm{a}} \mathrm{V}_{\mathrm{L}}$, and $\mathrm{V}_{2}$ through $\mathrm{V}_{6}$ leads by $0.2-0.4 \mathrm{mV}$. 
drome. Cineangiography showed no stenosis or obstruction in the right (data not shown) and left coronary arteries (left panel, Figure 2). Left ventriculography revealed akinesis in the apex, and severe asynergy in its surrounding area (right panel, Figure 2). Chest pain was relieved by intracoronary infusion of nitroglycerin and nicorandil, but continued to be mild for the next few hours. Creatine kinase $(214 \mathrm{IU} / \mathrm{L})$ and C-reactive protein $(0.5 \mathrm{mg} / \mathrm{dL})$ levels were mildly elevated 12 hours after admission. On the third hospital day, echocardiography documented the absence of wall motion abnormality. On ECG, ST elevation turned to deep negative $\mathrm{T}$, but $\mathrm{R}$ waves did not decrease.

Since stenotic, spastic, or occlusive sites were not found in epicardial coronary arteries when the chest pain was lasting, we suspected coronary microvasculature involvement in the pathophysiology of this event. The patient received the second catheterization 4 weeks later. In this catheterization, there was no significant vasospasm in the epicardial coronary arteries due to the two to three doses of intracoronary acetylcholine $(25,50$ and $100 \mu \mathrm{g}$ for the left coronary artery, 25 and $50 \mu \mathrm{g}$ for the right coronary artery, administration for 30 seconds each). Coronary microvascular function was then assessed. First, a Doppler flow wire was placed in the proximal portion of the large high lateral branch of the left coronary artery to measure the average peak flow velocity [APFV $(\mathrm{cm} / \mathrm{sec})]$ of the coronary artery. Second, by intracoronary nitroglycerin ( $2.5 \mathrm{mg}$ bolus) administration, the epicardial portion of the left coronary artery was kept maximally dilated, following which acetylcholine ( $50 \mu \mathrm{g}$ for $30 \mathrm{sec}$ ) was again administered into the coronary artery through the same guiding catheter. Thus, the change in APFV in response to intracoronary acetylcholine (IC-ACh) was considered to reflect the coronary microvascular response to it. In the nonischemic control subjects with

\section{Cineangiography of the left coronary artery End-diastole End-systole}
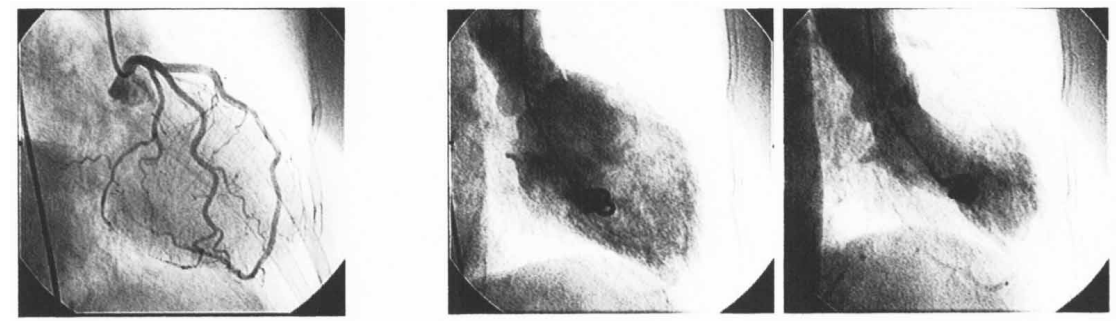

Figure 2. Cineangiography and left ventriculography on admission.

Cineangiography showed no stenosis or obstruction in the right (data not shown) and left coronary arteries (left panel). Left ventriculography documented akinesis in the apex and severe asynergy in its surrounding area. The area of such wall motion abnormality did not match the perfusion area of the left or right coronary artery anatomically. 
chest pain $(n=24)$, we obtained data showing that basal APFV increased to 296 $\pm 29 \%$ of the basal value after IC-ACh (left panel, Figure 3). In this patient, although acetylcholine administration did not change the systemic blood pressure or heart rate, and cineangiography did not document vasospasm in epicardial coronary arteries, APFV decreased to $54 \%$ of its basal value (right upper panel, Figure 3). Since these cardiac catheterization data suggested the involvement of coronary microvascular spasm, she was prescribed nifedipine (slow releasing type) $40 \mathrm{mg} /$ day and nicorandil $15 \mathrm{mg} /$ day. Although therapeutic prevention by drug administration for coronary microcirculatory impairment was not confirmed because she did not agree with the third catheterization while taking the medicine, she tolerated the drugs well, had no cardiac symptoms after that, and was discharged from our hospital.

She was followed-up with medical treatment in our outpatient clinic and no chest pain attacks were reported. However, in September 2003, she was admitted to our emergency room because of severe chest pain mimicking acute myocardial infarction like she experienced in 1999. It was found that she had forgotten to take

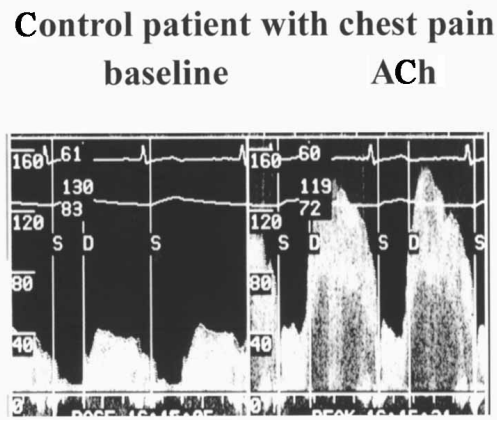

This patient baseline ACh (without medicine, 1999)

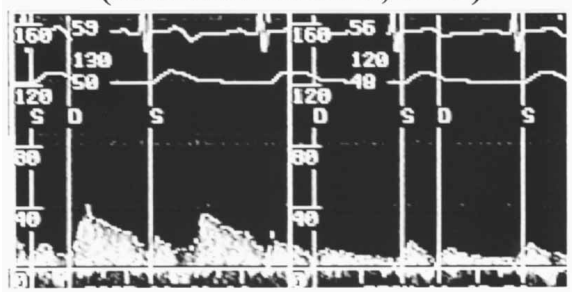

(with medicine, 2003)

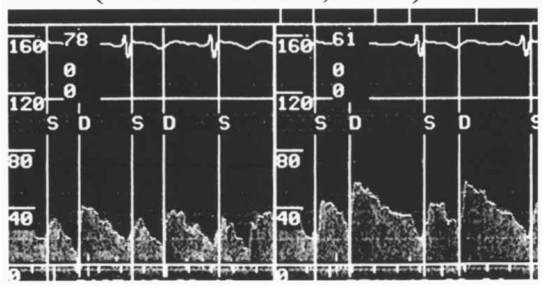

Figure 3. Doppler flow pattern of a nonischemic subject (left panel: control pattern with chest pain) and this patient (right panels).

In the nonischemic control subject, under maximal dilatation of the epicardial coronary artery by intracoronary nitroglycerin administration, basal average peak flow velocity $(\mathrm{cm} / \mathrm{sec})$ increased to $296 \pm 29 \%(n=24)$ by intracoronary acetylcholine (ACh) (representative data in the left panel). In this patient 4 weeks after the first cardiac event in 1999 ('without medicine, 1999' in the right upper panel), the average peak flow velocity measured by the Doppler flow wire method was decreased to $54 \%$ of its baseline value. In this patient 3 weeks after the second cardiac event in 2003 ('with medicine, 2003' in the right lower panel), the average peak flow velocity increased to $177 \%$ of the basal value. 
her medicine for the past 3 days. Emergency cardiac catheterization (third catheterization for her) documented left ventricular apical ballooning on a left ventriculogram. However, neither coronary stenosis nor occlusion were revealed on cineangiograms, as in the case in 1999. Left ventricular endocardial myocardial biopsy revealed no evidence of acute myocarditis. Since these findings suggested that the transient diminished drug effect was the trigger of the recurrence of the attack, medical treatment with nefedipine and nicorandil at the doses mentioned above was started again, and she had no chest pain attacks thereafter. While continuing these medications, 3 weeks after the second attack, she underwent a fourth cardiac catheterization to assess whether the coronary microcirculatory spasm could be prevented by these drugs. Her APFV under pretreatment with intracoronary nitroglycerin increased to $177 \%$ of its basal value by intracoronary acethylcholine administration to the LCA. Thus, transient coronary microvascular spasm, which was supposed to have been induced by acethylcholine in the second catheterization in 1999, appeared to be prevented under the drug regimen. Although the increase in coronary flow reserve seemed to be still lower than that in the nonischemic control subjects as shown above, and since the medicines taken were all at maximal doses and her chest pain attacks were resolved by these drugs, she was discharged and is currently continuing these medications.

It is known that coronary microvascular spasm can mimic ischemic heart disease. ${ }^{9)}$ In the present study, intracoronary administration of acetylcholine under vasodilatation of the epicardial coronary artery with nitroglycerin reduced APFV without evoking epicardial coronary spasm, and without significant changes in cardiac demand or coronary perfusion pressure, suggesting that flow reduction in this coronary perfusion bed was due to coronary microvascular constriction by acetylcholine. The present patient had a recurrent attack 4 years after the first one. The second attack seems to be triggered by a failure of the patient to take her medication as directed. As shown by the results of the fourth catheterization, daily oral $\mathrm{Ca}$ antagonist and ATP-sensitive $\mathrm{K}$ channel opener administration at the maximal doses was likely to prevent the occurrence of acethylcholineinduced coronary microvasular spasm. Although it still remains to be determined whether this finding is common in the pathogeny of this syndrome, in this patient coronary microvascular spasm was thought to be involved in the pathophysiology of reversible ventricular dysfunction which mimicked acute coronary syndrome due to epicardial coronary artery disease.

In addition, coronary flow reserve was still low in this patient after restarting the medications compared to the nonischemic control subjects as mentioned above, and this patient's clinical course needs to be followed-up in the long-term carefully to assess whether the present medical treatments are adequate for managing this microvascular disorder. 


\section{REFERENCES}

1. Shaw TR, Rafferty P, Tait GW. Transient shock and myocardial impairment caused by phaeochromocytoma crisis. Br Heart J 1987; 57: 194-8.

2. Kawai S, Suzuki H, Yamaguchi H, et al. Ampulla cardiomyopathy ('Takotsubo' cardiomyopathy) - reversible left ventricular dysfunction with ST segment elevation. Jpn Circ J 2000; 64: 156-9.

3. Owa M, Aizawa K, Urasawa N, et al. Emotional stress-induced 'ampulla cardiomyopathy': discrepancy between the metabolic and sympathetic innervation imaging performed during the recovery course. Jpn Circ J 2001; 65: 349-52.

4. Tsuchihashi K, Ueshima K, Uchida T, et al. Transient left ventricular apical ballooning without coronary artery stenosis: a novel heart syndrome mimicking acute myocardial infarction. J Am Coll Cardiol 2001; 38: 11-8.

5. Kurisu S, Sato H, Kawagoe T, et al. Tako-tsubo-like left ventricular dysfunction with ST-segment elevation: a novel cardiac syndrome mimicking acute myocardial infarction. Am Heart J 2002; 143: 448-55.

6. Kurisu S, Inoue I, Kawagoe T, et al. Time course of electrocardiographic changes in patients with tako-tsubo syndrome: comparison with acute myocardial infarction with minimal enzymatic release. Circ J 2004; 68: 7781.

7. Kume T, Akasaka T, Kawamoto T, et al. Relationship between coronary flow reserve and recovery of regional left ventricular function in patients with tako-tsubo-like transient left ventricular dysfunction. J Cardiol 2004; 43: 123-9. (Japanese)

8. Kyuma M, Tsuchihashi K, Shinshi Y, et al. Effect of intravenous propranolol on left ventricular apical ballooning without coronary artery stenosis (ampulla cardiomyopathy): three cases. Circ J 2002; 66: 1181-4.

9. Mohri M, Koyanagi M, Egashira K, et al. Angina pectoris caused by coronary microvascular spasm. Lancet 1998; 351: 1165-9. 